\title{
Correction: The AURORA Study: a longitudinal, multimodal library of brain biology and function after traumatic stress exposure
}

Samuel A. McLean (1) - Kerry Ressler • Karestan Chase Koenen - Thomas Neylan • Laura Germine - Tanja Jovanovic • Gari D. Clifford · Donglin Zeng • Xinming An · Sarah Linnstaedt 1 - Francesca Beaudoin · Stacey House • Kenneth A. Bollen · Paul Musey · Phyllis Hendry · Christopher W. Jones · Christopher Lewandowski • Robert Swor • Elizabeth Datner - Kamran Mohiuddin - Jennifer S. Stevens (D) Alan Storrow - Michael Christopher Kurz • Meghan E. McGrath · Gregory J. Fermann · Lauren A. Hudak · Nina Gentile · Anna Marie Chang • David A. Peak • Jose L. Pascual $(\mathbb{D} \cdot$ Mark J. Seamon - Paulina Sergot - W. Frank Peacock - Deborah Diercks - Leon D. Sanchez • Niels Rathlev · Robert Domeier · John Patrick Haran • Claire Pearson • Vishnu P. Murty · Thomas R. Insel • Paul Dagum - Jukka-Pekka Onnela - Steven E. Bruce • Bradley N. Gaynes • Jutta Joormann - Mark W. Miller (1) Robert H. Pietrzak - Daniel J. Buysse - Diego A. Pizzagalli (1) - Scott L. Rauch - Steven E. Harte • Larry J. Young • Deanna M. Barch $(1) \cdot$ Lauren A. M. Lebois (1) - Sanne J. H. van Rooij $(1)$ Beatriz Luna - Jordan W. Smoller · Robert F. Dougherty - Thaddeus W. W. Pace - Elisabeth Binder (1) John F. Sheridan - James M. Elliott Archana Basu (1) - Menachem Fromer (1) - Tushar Parlikar · Alan M. Zaslavsky · Ronald Kessler (1)

Published online: 28 September 2020

(c) Springer Nature Limited 2020

Correction to: Molecular Psychiatry (2020) 25:283-296 https://doi.org/10.1038/s41380-019-0581-3 published online 19 November 2019

Following publication of this article, the authors noticed that the word "not" had been accidentally omitted from the sentence: "A similar pattern is found in the military, where the great majority of APNS cases are found among those who are not severely injured". This has been corrected in both the PDF and HTML versions of this article. 